\title{
The Link Between Brain and Auditory System and Possible Role of Vestibular System in Hearing System as a Second Middle Ear and the Role of Melanocytes and/or Neuromelanin in this Process
}

\author{
Alireza Bina* \\ Starwood Audiology, USA
}

*Corresponding author: Alireza Bina, Starwood Audiology, USA,

Email: abina@starwoodaudiology.net

\begin{abstract}
Backward Auditory input pathways of Sound hypothesis describes that sound might be transferred from our Skin and eyes to the Inner Ear and the External Auditory Canal is not the only input of Sound, The Cochlea receives the sound from two separate directions Forward Auditory input pathway which are the Signals reach out to the cochlea from the External ear canal and the second direction Backward Auditory input pathways which are the signals transfer from our Skin and eyes and approach to the cochlea. The cochlea receives the sound from both directions and organizes and transfers them to the Brain for the ultimate processing.

We deal with this subject by explaining

A: General features of Melanocytes, Melanin and Neuromelanin

B: Those parts of the Brain contain Neuromelanin and their link with Vestibular system

C: Vestibular System, Third Window Disorders and Third Window Mobile Hypothesis vs second middle ear disorders hypothesis

D: Possible implications of Vestibular system and Neuromelanin/Melanin in Backward Auditory Input pathways of sound
\end{abstract}

Keywords: Otosclerosis; Meniere's syndrome; Forward and backward auditory input pathways; Melanin; Neuromelanin; Melanocytes; Third window disorders; Vestibular System; Sensorineural hearing loss; Cochlea; Conductive hearing loss; Semicircular canals; Utricle; Saccule; Round Window; Oval Window

\section{Melanocytes and Neuromelanin}

Skin's Melanocytes are situated on the Stratum Basale of the skin's epidermis and they are considered melanin-producing neural crest-extracted cells. They are also all over the body such as the uvea which is the middle layer of the Eye, the Cochlea and Vestibular System, vaginal epithelium, Leptomeninges, bones, and heart [1,2]. Melanin is a dim pigment and one of its roles is in Skin color. When the Melanin is synthesized. It is included in a specific multinucleate so called melanosomes which is delivered to adjacent keratinocytes to produce pigmentation. Melanocytes and Melanin have a function in the immune system also protection against UV radiation and many other roles [3,4]. Melanocytes generate melanin through a procedure called melanogenesis, this pigment is noticed in many parts of the body. The pigmentation which initiates from Oxidation of already existing Melanin is different from this 
Melanogenesis result to pigmentation [5]. Melanogenesis contains basal and actuated levels; low basal levels of melanogenesis are usually seen more in people with brighter skin's color. Elevation in melanogenesis has been observed in exposure to UV-B radiation. Physiologically melanogenesis protects the hypodermis, which is the layer under the skin, from damage by UV-B radiation. The Melanin absorbs the UV-B light by its dark color and does not let it passes through the Epidermis [6]. In a square millimeter of skin almost 1000 to 2000 melanocytes could be found or almost five to ten percent of the cells in the deepest part of the epidermis. The length of the Melanocytes is usually 7 Micrometer, however, their size can be diverse $[7,8]$. The amount of the Melanocytes is not in charge of the skin color between dark and brighter skin people, but the level of the quantity of the melanocytes also amounts of eumelanin and pheomelanin is involved in this procedure [9].

Melanocytes are believed to have a function in Immune system, and they are considered sort of Immune cells however their function in Immune system is not completely understood so far [10]. Protecting the body by ingesting harmful foreign particles, branched feature look like, Cytokines producing are some of the same features between Melanocytes and Dendritic cells [11]. albeit both melanocytes and Dendritic cell have some similarities, but they are not completely identical. The cell lineages which they are derived from are completely different [11]. Presenting antigen and producing Cytokine are some of the function of melanocytes in immune system [12-14].

IL-1, IL-3, IL-6, IL-8, TNF- $\alpha$, and TGF- $\beta$ are Proinflammatory cytokines which can be produced by melanocytes [15]. Besides, cytokines discharged by other nearby immune cells stimulate the cytokine's production by melanocytes [15]. Melanocytes are the protector's cells against harmful pathogens. Melanocytes utilize their dendrites to communicate with cells in other layers other than the Stratum basale [17]. Melanocytes fight against any pathogens which come to the epidermis [17]. Melanocytes are in link with both Keratinocytes and Langerhans cells which both are strongly phagocytic in response to the immune system.

It is observed that Melanogenesis could be changed by numerous stimuli, albeit this process and how it works is unknown right now. The Melanoblast is the Melanocyte precursor's cell. Stem cells are located in the projection section of the external deepest part of hair follicles in adults. The stem cells are actuated when a hair is lost and regrows again. Both keratinocyte precursors and melanoblasts are the transformation products of these stem cells and they enrich both hair and skin. There is also proof that in cutaneous nerves Melanocytes stem cells are existing, with nerve signals resulting them to convert into melanocytes for the skin [17].

\section{Neuromelanin}

Neuromelanin (NM) is a dim pigment that exists in some parts of the brain that is mainly associated to melanin. The substantia nigra pars compacta, locus coeruleus, ventrolateral Reticular
Formation Medulla Oblongata are those parts of the brain which NM can be found in large amounts [17]. Neuromelanin provides typical brain sections, which mentioned earlier definite color. It is a branch of melanin and looks like the other types of melanin which is found in peripheral sections. It is insoluble in organic consolidations, also it can be characterized by silver staining. The name of the Neuromelanin is because of its role also the feature of color's changes that emerge in those substances which contain the NM. NM carries dark and hazel pigmented spots. NM is seen to amass in aging, considerably after the first 2 or 3 years of life. The reason of this is probably because of the protection role of NM against iron- induced oxidative stress in Substantia Nigra $[18,19]$.

Neuromelanin is observed in more quantities in humans compared to non-human primates [17]. The concentration of the Neuromelanin is increased with the aging process probably because of the protection of the neurons. It is believed that in Parkinson disease neurodegeneration of the Neuromelanin which contains some Neurons and cell death in substantia nigra is involved in this disease. Probably Oxidative stress in this region is the reason for the cell death and supposedly the motor symptoms in this disease is because of this Oxidative stress. The Neuromelanin might be able to revive this Oxidation. The quantity of the Neuromelanin of Substantia Nigra is usually 50 percent less in patients who suffer from Parkinson disease compared to normal individuals. The Neurodegeneration of the Neuromelanin in Pars Compacta and Locus Coeruleus also involved in this disease and has been proved and observed by imaging of the Neuromelanin on these parts of the Brain [18]. Neuromelanin could prevent the Neurodegeneration by binding neurotoxic and/or toxic metals [18].

Purkyně was the first scientist who expressed black pigments in Substantia Nigra in 1838 and Lillie described the Neuromelanin in 1957 and on that time they believed that Neuromelanin physiologically has no role but now it is scientifically proved that they are cell protectors of some parts of the Brain also their link with Parkinson disease and because of this possibility scientist strongly are doing research in Neuromelanin [19]. Melanocytes and Neuromelanin also have the ability to absorb the mechanical energy such as Sound and light and transform the light to sound and vice versa. They have an important function in hearing system which will be explained later in this paper [20-29].

\section{Neuromelanin-Locus coeruleus}

The posterior area of the rostral pons in the lateral floor of the fourth ventricle is a section of the brain which Locus Coeruleus (LC) is located. It is in the brainstem and a branch of the Reticular activating system. One of its functions is controlling the panic and Stress [30].

LC is the major source of the Norepinephrine along with adrenal medulla which supplies the Norepinephrine directly to the blood [31]. Medium size Neurons could be found in LC. The color of the LC is blue and it is because of the Melanin or pigmented 
cells inside the neurons of the LC. LC is a nucleus in the pons and is strongly pigmented and because of this feature another name of the LC is the Nucleus Pigmentosus Points. The Neuromelanin of the LC is similar to the dark Neuromelanin dopamine base of the Substantia Nigra and Polymerization of noradrenaline is structured the Neuromelanin of the LC [32]. The quantities of the pigmented neurons in LC are almost twenty two thousands to fifty one thousands and their size is different from 31,000 and $60,000 \mu \mathrm{m} 3$. This is apply to humans between the age of 19 to 79 years old [33].

The LC has many projections to many parts of the brain. The LC is involved in Arousal and sleep awake cycle by its excitatory effect of the Neuromelanin $[33,35]$. Some other functions of the LC are the homeostatic control center of the body, Attention, memory, behavioral and/or cognitive elasticity, creativity, cognitive control, neuroplasticity, emotions, posture and balance and so on. Since the LC is a branch of reticular activating system then it is inactivated during rapid eye movement stage of the sleep [34,36-38].

It is believed that there is a link between the LC and many pathologies such as Alzheimer, Parkinson, depression and so on [35]. There is a high possibility of the link between Norepinephrine dysfunction of the LC and many cognitive and neuropsychiatric disorders such as PTSD, ADHD or other cognitive disorders which mentioned earlier [36,37]. The LC is also involved in some other disorders as well such as progressive supranuclear palsy, Pick's disease, Down syndrome [38]. The Locus Coeruleus has a tremendous role in Audio/vestibular System, The LC likely standby the Cochlea and/ or Vestibular system during sleep by its inactivation through Reticular Formation and Reactivate it again during arousal. LC and its connections are responsible for Pupil dilation following Auditory Stimuli. Many studies have been done recently regarding Pupil dilation following Auditory stimuli which can be used for the diagnosis of the Hidden Hearing Loss (cochlear Synaptopathy) also it can be used for the diagnosis of the hearing loss as an objective test very Soon [39].

\section{Neuromelanin, Substantia Nigra- Pars Compacta}

The Midbrain is a branch of the Brain which Substantia nigra (SN) is located and Pars Compacta (PC) is a part of SN so it is called Substantia Nigra Pars Compacta (SNPC) The SNPC contains Dopaminergic neurons and the dark pigmented Neuromelanin are existed in this section of the brain. The neurons of the SNPC are not involved in movement directly but it organizes the Striatum and by this management role it is involved in movement indirectly so dysfunction on Pars Compacta neurons have a huge impact on movement which can be seen in patients who suffer from Parkinson disease [40]. Parkinson disease could be sometimes genetic or secondary to infections or toxins [41]. Dysfunction on this section of the brain may also involve schizophrenia and/or addictive conduct as this part of the brain is involved in reward and pleasure as well. Spatial learning and temporal processing, inspection of the environment and location in space also part of the memory, sleep wake cycle are other functions of the PC [41-44]. Substantia Nigra is a branch of Basal Ganglia and it has a tremendous role in the Hearing and Balance system. Basal Ganglia implicated in speech categorization and speech recognition. Patients who suffer from sensorineural hearing loss and also have trouble understanding nonnative accent likely have dysfunction in Basal Ganglia. Vestibular System is probably involved in Parkinson Disease as well because of its link and connections with Substantia Nigra $[41,42,43,46]$.

\section{Neuromelanin- Ventrolateral Reticular formation- Medulla Oblongata}

The reticular formation is a region of the Brainstem and its neurons located from top of the Midbrain to the bottom section of the Medulla Oblongata [40-42]. Somatic motor control, maintaining tone, balance, and posture typically during body movements, imparts eye and ear signals to the cerebellum so that the cerebellum can combine visual, auditory, and/or vestibular stimuli in motor coordination. Activation the eyes to trace and fixate objects, modulation of breathing and swallowing, Cardiovascular control, modulation of the pain. Sleep and states of consciousness such as alertness and sleep, Habituation are some physiological functions of the Reticular Formation [42-51].

\section{Medulla Oblongata}

The medulla oblongata or medulla is a section of the brainstem [52]. It is located near to the Cerebellum. (Anterior-inferior part of the Cerebellum) [53]. Physiologically it is in charge of Vomiting, Sneezing, coughing, swallowing. Cardiac, respiratory, breathing, heart rate, blood pressure, sleep wake cycle [54-58].

There is a significant link between Medulla and the Audio / vestibular system. Vomiting following Vestibular dysfunction also increasing blood pressure following Vestibular loss are perhaps because of the link between Medulla with Audio -Vestibular system. We have seen many patients during our clinical experience without having any history of blood pressure which their blood pressure increased following Vestibular disorders although other reasons is proposed for this such as hypertension following stress secondary to Vertigo but now a days the function of Vestibular system in regulating blood pressure is observed [59].

\section{Meninges- Leptomeninges}

The meninges consist of three different sections in human, dura mater, the arachnoid mater and the pia mater. Between the arachnoid mater and pia mater which is the subarachnoid space the Cerebrospinal fluid could be observed. Meninges function is to protect the Central Nervous system. So-called Leptomeninges are arachnoid and pia mater together [60]. The arachnoid mater or arachnoid membrane is the middle part of the Meninges, it is called Arachnoid membrane because it looks like a spider web. It is consisted of fibrous tissue as well as the pia mater, has an external covering of firmly filled prone cells, establishing the arachnoid barrier [61]. Arachnoid Trabeculae is a complex of filament which 
proceed from the arachnoid through the subarachnoid space to combine with the pia mater. There is a collagen on exterior side of arachnoid barrier which is responsible for essential meningeal barrier across the cerebrospinal fluid and subarachnoid space as well as the blood circulation in the dura [62]. The pia mater is a sensitive and narrow membrane. It covers the meningeal section of the brain and tightly attach to the brain's exterior part as well as spinal cord. It is located following the gyri and sulci. It consists of fibrous tissue extended on its external layer by some cells believed to be impervious to fluid. It is believed that Leptomeninges produces the beta-trace protein or prostaglandin D2 synthase, which is the main cerebrospinal fluid protein. The Leptomeninges are in connection with Semicircular canals through the CSF and /or Perilymph via Melanocytes which can be found in Leptomeninges as well as Semicircular Canals in the Vestibular system [63-70].

\section{Vestibular system, Third Window Disorders and Third Window Mobile Hypothesis vs Second Middle Ear Disorders Hypothesis}

The vestibular system is physiologically involved in balance and /or spatial detection. It contains two different sections, the first section is Semicircular canals (Superior, posterior, horizontal) Superior and posterior canals are considered vertical semicircular canals. The lateral canals are involved in head's rotation on vertical axis for example during gyrations, on the other hand the Superior and posterior canals are involved in longitudinal and/or coronal plane rotations for example during nodding or circular handspring. The second section of the Vestibular system is Otoliths (Utricle, Saccule) which is in charge of linear acceleration [71,72]. The muscles receive the signal from Vestibular system which give us the ability to upright and control the posture [73-86].

The link between the brain and Vestibular system lead to the comprehension of the body's dynamic and/or Kinematics which eventually helps us to figure out the position and/or acceleration from time to time [76].

Studies showed both Semicircular canals and Otolith Organs are involved in Hearing system as well, Sudden deafness following Benign paroxysmal positional vertigo (BPPV), Abnormal Video Head Impulse test and Caloric test in Idiopathic Sudden Deafness, Otolith dysfunction in High Frequencies hearing loss and Profound Sensorineural Hearing loss are some examples of their significant link with Auditory system [87-89].

Third Window Disorders are referred to those pathologies of the Inner ear which typically cause Conductive or Mixed Hearing loss, in normal ears there are two Widows, Round and Oval Window. According to the Third Window mobile hypothesis the Third window caused by these pathologies such as Superior, Posterior, Lateral Semicircular Canals dehiscence, Large Vestibular Aqueduct Syndrome and so on is the reason for the Conductive hearing loss on these disorders. in consonance with this hypothesis because of abnormal Third Window caused by these pathologies transmission of the sounds from the Oval Window to the Round Window is disrupted and acoustic energy cannot reach out to the Round window properly and eventually less sound energy accessible to the hair cells [90-93]. If Third Window Mobile hypothesis is completely true then we should expect less Air bone gap ( Conductive Hearing Loss) in concomitant Superior Semicircular Canal dehiscence (SSCD) and Otosclerosis compare to patients who suffer from SSCD only because the stiffness effect following Otosclerosis conquer the Hyper mobility induced by the Third Window but most case reports in Concomitant SSCD and Otosclerosis showed maximum Air Bone Gap also the Round Window reinforcement surgery technic must make the hearing loss lessen or cure but studies showed this surgery technique can make the symptoms alleviated but the hearing loss following SSCD could not be changed after surgery and even Round window complete occlusion technic causes the situation worse in some case studies. It seems that physiologically the Vestibular system is the second middle ear and Vestibular system receives the auditory information as well through the engagement between Vestibular Melanocytes and CNS Melanocytes (Melanin, Neuromelanin) and delivers the information to the cochlea. (Second Middle ear disorders hypothesis) [93-97].

\section{Discussion}

The Brain Functional Imaging studies such as fMRI and Functional Near-infrared spectroscopy (FNIRS) showed that the Hearing or Auditory system is a Multimodal system [98]. Vision/ eyes and, Tactile/skin are involved in the hearing system [98,99]. Echolocation in some animals and humans also Gesturelocation (ability of some blindness people to do the gesture and move their hand frequently to specific directions and identify the objects size and shape) are some examples of the link between these senses with each other $[123,124]$. It seems that Utricle and Saccule receive the auditory information from two different directions forward auditory input pathway and Backward Auditory input pathways [100]. In forward auditory input pathway auditory signals come to the external ear canal, Tympanic Membrane, Ossicles and eventually Utricle and Saccule and in backward Auditory input pathways of sound signals collected from skin/Tactile and vision/eyes by Central Nervous System (CNS) Melanocytes and Neuromelanin. The CNS Melanocytes are in connection with Vestibular and/or Cochlea's Melanocytes. The Neuromelanin of the locus coeruleus, Pars Compacta, Ventrolateral Reticular formationMedulla Oblongata, Leptomeninges and their connections are involved in transmission of the sound from Skin/tactile and Vision/ eyes to the Semicircular Canals and eventually Utricle and Saccule. In the backward auditory input pathway of sound, there is a high possibility that Melanocytes and Neuromelanin pass the auditory information through Gap Junctions [107]. In Utricle and Saccule the auditory information will be separated from Vestibular information and the auditory information from two different directions (forward auditory input pathway and backward auditory input pathways) will be combined and eventually the combined information will be 
delivered it to the cochlea [100-106]. Melanin and pigmented cells have been observed around the Round Window Membrane (RW) [108]. The auditory signals which come through the forward and backward auditory input pathways absorb by RW membrane by its outward movement following the vibration of the oval window also its pigmented cells and conduct them to the cochlea. The Round Window pumps all the combined auditory information from two directions (forward and backward auditory input pathways) and delivers it to the Cochlea [108]. both cochlear conductive hearing loss and Sensorineural hearing loss have been reported in RW Pathologies. Type of the hearing loss depends on the site and degree of the lesion in RW membrane and its surrounding $[109,110]$. Cochlear conductive hearing loss also has been reported in Meniere's disease, although another hypothesis is proposed but the Vestibular organs dysfunction because of stiffness caused by hydrops might be the reason for the conductive component hearing loss in this disease [111]. Albeit Conductive or Mixed Hearing loss is more common in Superior, posterior, lateral Semicircular Canals Dehiscence and Large Vestibular Aqueduct Syndrome but Sensorineural hearing loss has been reported as well so type of the hearing loss depends on the site and degree of the lesion as Vestibular system contains pigmented and transitional cells [112115]. Pupil Dilation following auditory stimuli has been observed [116]. Ocular and CNS Melanin whether absorb the Sound as well as light and transfer it to the Semicircular canals, Utricle and Saccule and eventually the cochlea or Ocular and CNS Melanin transform the sound to the light and then transmit the light to the Semicircular Canals, Utricle and Saccule and eventually the cochlea and sense of hearing occurs following Optogenetic like stimulation and/ or genetic engineering like $[117,118]$. It seems that Semicircular Canals have the ability to detect the sound from different directions through the connection of their Melanocytes and CNS melanocytes and act as an Antenna. In some animals like Cheetah, which is one of the fastest creature on earth, the Semicircular Canals are much larger than human as well as more variety of Melanocytes which give the ability to detect any movement of other creatures also the amazing speed and balance system and ability to zoom precisely on the target $[119,120]$.

\section{Conclusion}

Engagement of the subcortical area of the brain which mentioned earlier in backward auditory input pathways of sound is more prominent than cortical areas. It seems that the subcortical areas of the CNS are the coordinators of the hearing system between Peripheral sections and cortical areas. It is likely that part of the Vestibular system physiologically is responsible for transmitting part of the auditory information to the Cochlea. Melanocytes all over the body are in link and connection with each other and this is one of the reasons or hypotheses of the hearing loss in renal failure and/or Hearing loss in heart disorders [121,122]. Investigations and research regarding the Melanocytes and Neuromelanin not only open the new doors for the treatment of hearing loss, it can open the doors for the treatment of many other disorders as they are all over the body also they are in link with many Pathologies. The Vestibular system has a tremendous role in the auditory/ Hearing system. The Cochlea and Vestibular system are actually one system that work together. There is no sense of hearing without Vestibular system and Vice Versa. It seems that the Cochlea receives the sound from two different directions, Forward auditory input pathway and backward auditory input pathways. The Cochlea is the ultimate target of all the auditory information from both forward and backward auditory input pathways of sound and after initial coding and processing it sends the information to the Brain for the terminal processing [123-125].

\section{Acknowledgments}

None.

\section{Conflicts of Interest}

The author declares that there is no conflict of interest to disclose.

\section{Funding}

None.

\section{References}

1. Cramer SF (1991) The origin of epidermal melanocytes. Implications for the histogenesis of nevi and melanomas". Archives of Pathology \& Laboratory Medicine 115 (2): 115-119.

2. Stefán AH Gudjohnsen, Diahann AM Atacho, Franck Gesbert, Graca Raposo, Ilse Hurbain, et al. (2015) Meningeal Melanocytes in the Mouse: Distribution and Dependence on Mitf. Front. Neuroanat 9: 149.

3. Markert CL, Silvers WK (1956) The Effects of Genotype and Cell Environment on Melanoblast Differentiation in the House Mouse. Genetics 41(3):429-50.

4. Mayeaux EJ, Cox JT, et al. (American Society for Colposcopy and Cervical Pathology) (2011-12-28). Modern Colposcopy Textbook and Atlas. Lippincott Williams \& Wilkins.

5. Mintz B (1971) Clonal basis of mammalian differentiation. Symp Soc Exp Biol 25:345-70.

6. NICHOLS SE, REAMS WM (1960) The occurrence and morphogenesis of melanocytes in the connective tissues of the PET/MCV mouse strain. J Embryol Exp Morphol 8: 24-32.

7. Theriault LL, Hurley LS (1970) Ultrastructure of developing melanosomes in C57 black and pallid mice. Dev Biol 23(2): 261-275.

8. Agar N, Young AR (2005) Melanogenesis: a photoprotective response to DNA damage? Mutat Res 571(1-2): 121-32.

9. Parrish JA, Jaenicke KF, Anderson RR (1982) Erythema and melanogenesis action spectra of normal human skin. Photochem Photobiol 36(2): 187-91.

10. Montoliu L, Grønskov K, Wei AH, Martínez-García M, Fernández A, et al. (2014) Increasing the complexity: new genes and new types of albinism. Pigment Cell Melanoma Res 27(1): 11-8.

11. Gasque P, Jaffar-Bandjee MC (2015) The immunology and inflammatory responses of human melanocytes in infectious diseases. J Infect 71(4): 413-421.

12. Abdallah F, Mijouin L, Pichon C (2017) "Skin Immune Landscape: Inside and Outside the Organism”. Mediators of Inflammation: 5095293. 
13. Tapia CV, Falconer M, Tempio F, Falcón F, López M, et al. (2014) Melanocytes and melanin represent a first line of innate immunity against Candida albicans. Med Mycol 52(5): 445-454.

14. Roméro-Graillet C, Aberdam E, Biagoli N, Massabni W, Ortonne JP, et al (1996) Ultraviolet $B$ radiation acts through the nitric oxide and cGMP signal transduction pathway to stimulate melanogenesis in human melanocytes. J Biol Chem 271(45): 28052-28056.

15. Erin M Wolf Horrell, Mary C Boulanger, John A D’Orazio (2016) Melanocortin 1 Receptor: Structure, Function, and Regulation. Front Genet 7: 95.

16. Eller MS, Maeda T, Magnoni C, Atwal D, Gilchrest BA (1997) Enhancement of DNA repair in human skin cells by thymidine dinucleotides: evidence for a p53-mediated mammalian SOS response. Proceedings of the National Academy of Sciences of the United States of America 94(23): 12627-12632.

17. Cichorek M, Wachulska M, Stasiewicz A, Tymińska A (2013) Skin melanocytes: biology and development. Postepy Dermatologii I Alergologii 30 (1): 30-41.

18. Charkoudian LK, Franz KJ (2006) Fe(III)-coordination properties of neuromelanin components: 5,6-dihydroxyindole and 5,6-dihydroxyindole-2-carboxylic acid. Inorg Chem 45(9): 3657-3664.

19. Fedorow H, Tribl F, Halliday G, Gerlach M, Riederer P, et al. (2005) Neuromelanin in human dopamine neurons: comparison with peripheral melanins and relevance to Parkinson's disease. Prog Neurobiol 75(2): 10924

20. Rabey JM, Hefti F (1990) Neuromelanin synthesis in rat and human substantia nigra. J Neural Transm Park Dis Dement Sect 2(1):1-14.

21. Stepień K, Dzierzega-Lecznar A, Tam I (2007) Rola neuromelaniny w chorobie Parkinsona--nowe koncepcje The role of neuromelanin in Parkinson's disease--new concepts. Wiad Lek 60(11-12): 563-9.

22. Tribl F, Asan E, Arzberger T, Tatschner T, Langenfeld E, et al. (2009) Identification of L-ferritin in neuromelanin granules of the human substantia nigra: A targeted proteomics approach. Molecular \& Cellular Proteomics 8(8): 1832-1838.

23. Sasaki M, Shibata E, Tohyama K, Takahashi J, Otsuka K, et al. (2006) Neuromelanin magnetic resonance imaging of locus ceruleus and substantia nigra in Parkinson's disease. Neuroreport 17(11): 12151218.

24. Usunoff KG, Itzev DE, Ovtscharoff WA, Marani E (2002) Neuromelanin in the human brain: A review and atlas of pigmented cells in the substantia nigra. Archives of Physiology and Biochemistry 110 (4): 257-369.

25. Lillie RD (1957) Metal reduction reactions of the melanins: Histochemica studies. Journal of Histochemistry and Cytochemistry 5(4): 325-333.

26. Zecca L, Tampellini D, Gerlach M, Riederer P, Fariello RG, et al. (2001) Substantia nigraneuromelanin: Structure, synthesis, and molecular behaviour. Molecular Pathology 54 (6): 414-418.

27. Brash DE, Goncalves LCP, Bechara EJH (2018) Excited-State Medicine Working Group. Chemiexcitation and Its Implications for Disease. Trends Mol Med 24(6): 527-541.

28. Tolleson W.H. (2009) Melanin and Neuromelanin in the Nervous System. In: Binder M.D., Hirokawa N., Windhorst U. (eds) Encyclopedia of Neuroscience. Springer, Berlin, Heidelberg

29. Bernard R (1993) Ortiz De Montellano. Melanin, afrocentricity, and pseudoscience. American Journal of Physical Anthropology.

30. Mehler Mark F, Dominick P Purpura (2009) Autism, fever, epigenetics and the locus coeruleus. Brain Research Reviews 59 (2): 388-392.

31. Mouton PR, Pakkenberg B, Gundersen HJ, Price DL (1994) Absolute number and size of pigmented locus coeruleus neurons in young and aged individuals. J Chem Neuroanat 7(3): 185-90.

32. Benarroch EE (2009) The locus ceruleus norepinephrine system: functional organization and potential clinical significance. Neurology 73(20): 1699-1704.
33. Bouret S, Sara SJ (2005) Network reset: a simplified overarching theory of locus coeruleus noradrenaline function. Trends Neurosci 28(11): 574-582.

34. Aston-Jones Gary, Cohen Jonathan D (2005) An integrative theory of locus coeruleus-norepinephrine function: Adaptive gain and optimal performance. Annual Review of Neuroscience 28 (1): 403-450.

35. Schwartz JR, Roth T (2008) Neurophysiology of sleep and wakefulness: basic science and clinical implications. Current Neuropharmacology 6(4): 367-378.

36. Heneka MT, Nadrigny F, Regen T, Martinez-Hernandez A, DumitrescuOzimek L, et al. (2010) Locus ceruleus controls Alzheimer's disease pathology by modulating microglial functions through norepinephrine. Proc Natl Acad Sci U S A 107(13): 6058-6063.

37. Benarroch EE (2009) The locus ceruleus norepinephrine system: functional organization and potential clinical significance. Neurology 73(20): 1699-1704.

38. Sasaki M, Shibata E, Tohyama K, Takahashi J, Otsuka K, et al. (2006) Neuromelanin magnetic resonance imaging of locus ceruleus and substantia nigra in Parkinson's disease. Neuroreport 17(11): 12151218.

39. Costa VD, Rudebeck PH (2016) More than Meets the Eye: the Relationship between Pupil Size and Locus Coeruleus Activity. Neuron 89(1): 8-10.

40. Kim SJ, Sung JY, Um JW, Hattori N, Mizuno Y, et al. (2003) Parkin Cleaves Intracellular -Synuclein Inclusions via the Activation of Calpain. Journal of Biological Chemistry 278 (43): 41890-41899.

41. Francois C, Yelnik J, Tande D, Agid Y, Hirsch EC (1999) Dopaminergic cell group A8 in the monkey: anatomical organization and projections to the striatum. Journal of Comparative Neurology 414 (3): 334-347.

42. Langer LF, Jiménez-Castellanos J, Graybiel AM (1991) The substantia nigra and its relations with the striatum in the monkey. Prog Brain Res 87: 81-99.

43. Hajós M, Greenfield SA (1994) Synaptic connections between pars compacta and pars reticulata neurones: electrophysiological evidence for functional modules within the substantia nigra. Brain Res 660(2): 216-24.

44. Lavoie B, Smith Y, Parent A (1989) Dopaminergic innervation of the basal ganglia in the squirrel monkey as revealed by tyrosine hydroxylase immunohistochemistry. The Journal of Comparative Neurology 289(1): $36-52$

45. Pioli EY, Meissner W, Sohr R, Gross CE, Bezard E, et al. (2008) Differential behavioral effects of partial bilateral lesions of ventral tegmental area or substantia nigra pars compacta in rats. Neuroscience 153(4): 12131224

46. Lim SJ, Fiez JA, Holt LL (2014) How may the basal ganglia contribute to auditory categorization and speech perception? Front Neurosci 8: 230.

47. Antal M, Beneduce BM, Regehr WG (2014) The substantia nigra conveys target-dependent excitatory and inhibitory outputs from the basal ganglia to the thalamus. J Neurosci 34(23): 8032-8042.

48. Paul F Smith (2018) Vestibular Functions and Parkinson's Disease. Front Neurol 9: 1085

49. Dale Purves, George J Augustine, David Fitzpatrick, Lawrence C Katz, Anthony-Samuel LaMantia, James O McNamara, and S Mark Williams. Neuroscience, 2nd edition. Sunderland (MA): Sinauer Associates; 2001. ISBN-10: 0-87893-742-0

50. Hughes T (2003) "Neurology of swallowing and oral feeding disorders: Assessment and management". Journal of Neurology, Neurosurgery \& Psychiatry 74 (90003).

51. H Nishizawa, R Kishida, T Kadota, R C Goris, et al. (1988) Somatotopic organization of the primary sensory trigeminal neurons in the hagfish, Eptatretus burgeri. J Comp Neurol 267(2): 281-295.

52. Rovainen CM (1985) Respiratory bursts at the midline of the rostral medulla of the lamprey. J Comp Physiol A 157(3): 303-309. 
53. Brudzynski SM (2014) The ascending mesolimbic cholinergic system-a specific division of the reticular activating system involved in the initiation of negative emotional states. J Mol Neurosci 53(3): 436-445.

54. Kinomura S, Larsson J, Gulyás B, Roland PE (1996) Activation by attention of the human reticular formation and thalamic intralaminar nuclei. Science 271(5248): 512-515.

55. Kinomura S, Larsson J, Gulyás B, Roland PE (1996) Activation by attention of the human reticular formation and thalamic intralaminar nuclei. Science 271(5248): 512-515.

56. Tsoi SL, Orlov IV, Bovk TV (1991) Responses of frog vestibular neurons to combined temperature microstimulation of the semicircular canals. Neurosci Behav Physiol 21(2): 136-145.

57. Troiani D, Petrosini L, Zannoni B (1976) Relations of single semicircular canals to the pontine reticular formation. Arch Ital Biol 114(4): 337-375.

58. Jin GS, Li XL, Jin YZ, Kim MS, Park BR (2018) Role of peripheral vestibular receptors in the control of blood pressure following hypotension. Korean J Physiol Pharmacol 22(4): 363-368.

59. Tanya H Evans, Lawrence R Schiller (2012) Chronic vestibular dysfunction as an unappreciated cause of chronic nausea and vomiting Proc (Bayl Univ Med Cent) 25(3): 214-217.

60. Kumar, Vinay (2015) Robbins and Cotran Pathologic Mechanisms of Disease (9th ed.). Philadelphia: Elsevier Saunders. p. 1273. OCLC 892583347. In acute meningitis, an exudate is evident within the leptomeninges over the surface of the brain 28-21.

61. Abbott NJ, Patabendige AA, Dolman DE, Yusof SR, Begley DJ (2010) Structure and function of the blood-brain barrier. Neurobiol Dis 37(1): 13-25.

62. Yamashima Tetsumori, Sakuda Kazushige, Tohma Yasuo, Yamashita Junkoh, Oda Hiroshi, et al. (1997) Prostaglandin D Synthase ( $\beta$-Trace) in Human Arachnoid and Meningioma Cells: Roles as a Cell Marker or in Cerebrospinal Fluid Absorption, Tumorigenesis, and Calcification Process. Journal of Neuroscience 17 (7): 2376-2382.

63. van Gijn J, Kerr RS, Rinkel GJ (2007) Subarachnoid haemorrhage. Lancet 369(9558): 306-318

64. Lim ZM, Friedland PL, Boeddinghaus R, Thompson A, Rodrigues SJ, et al. (2012) Otitic meningitis, superior semicircular canal dehiscence, and encephalocele: a case series. Otol Neurotol 33(4): 610-612.

65. Maurizio Barbara, Valerio Margani, Anna Voltattorni, Simonetta Monini, Edoardo Covelli, et al. (2020) Concomitant Dehiscences of the Temporal Bone: A Case-Based Study. Ear, Nose\& Throat Journal, SAGE Journals.

66. Higuchi S, Sugahara F, Pascual-Anaya J, Takagi W, Oisi Y, et al. (2019) Inner ear development in cyclostomes and evolution of the vertebrate semicircular canals. Nature 565(7739): 347-350.

67. Barnett-Cowan M, Harris LR (2009) Perceived timing of vestibular stimulation relative to touch, light and sound. Exp Brain Res 198(2-3): 221-31.

68. Barnett-Cowan M (2013) Vestibular perception is slow: a review. Multisens Res 26(4): 387-403.

69. Rossmeisl JH (2010) Vestibular disease in dogs and cats. Vet Clin North Am Small Anim Pract 40(1): 81-100.

70. Smith Paul F, Darlington, Cynthia L (2013) Personality changes in patients with vestibular dysfunction". Frontiers in Human Neuroscience 7:678.

71. Necker R (2005) The structure and development of avian lumbosacral specializations of the vertebral canal and the spinal cord with special reference to a possible function as a sense organ of equilibrium. Anat Embryol (Berl) 210(1): 59-74.

72. Necker R (2006) Specializations in the lumbosacral vertebral canal and spinal cord of birds: evidence of a function as a sense organ which is involved in the control of walking. J Comp Physiol A Neuroethol Sens Neural Behav Physiol 192(5): 439-448.
73. Necker R, Janssen A, Beissenhirtz T (2000) Behavioral evidence of the role of lumbosacral anatomical specializations in pigeons in maintaining balance during terrestrial locomotion. J Comp Physiol A 186(4): 409412.

74. Angelaki DE, Cullen KE (2008) Vestibular system: the many facets of a multimodal sense. Annu Rev Neurosci 31: 125-150.

75. Chang TP, Newman-Toker DE (2019) The Link Between Bppv And Sudden Hearing Loss in Administrative Data-Biological Basis Or Diagnostic Deception? Otol Neurotol 40(6): 843-844.

76. Lee NH, Ban JH (2010) Is BPPV a Prognostic Factor in Idiopathic Sudden Sensory Hearing Loss? Clin Exp Otorhinolaryngol 3(4): 199-202.

77. El-Saied S, Joshua BZ, Segal N, Kraus M, Kaplan DM (2013) Sudden hearing loss with simultaneous posterior semicircular canal BPPV: possible etiology and clinical implications. Am J Otolaryngol. 2014 MarApr;35(2):180-185.

78. Youn Ho Kim, Dong Kuck Lee, Jung Im Seok (2011) Sudden Deafness Concurrent with Ipsilateral Benign Paroxysmal Positional Vertigo. Research in Vestibular Science10(2): 74-76.

79. Byun H, Chung JH, Lee SH (2020) Clinical implications of posterior semicircular canal function in idiopathic sudden sensorineural hearing loss. Sci Rep 10(1): 8313.

80. Yao Q, Xu C, Wang H, Shi H, Yu D (2018) Video head impulse test results suggest that different pathomechanisms underlie sudden sensorineural hearing loss with vertigo and vestibular neuritis: Our experience in fiftytwo patients. Clin Otolaryngol 43(6):1621-1624.

81. Sazgar AA, Dortaj V, Akrami K, Akrami S, Karimi Yazdi AR (2006) Saccular damage in patients with high-frequency sensorineural hearing loss. Eur Arch Otorhinolaryngol 263(7): 608-13.

82. Xu XD, Zhang Q, Hu J, Zhang Y, Chen YF, et al. (2015) The hidden loss of otolithic function in children with profound sensorineural hearing loss. Int J Pediatr Otorhinolaryngol 79(6): 852-857.

83. Tseng CC, Young YH (2013) Sequence of vestibular deficits in patients with noise-induced hearing loss. Eur Arch Otorhinolaryngol 270(7): 2021-2026.

84. Cushing SL, Gordon KA, Rutka JA, James AL, Papsin BC (2013) Vestibular end-organ dysfunction in children with sensorineural hearing loss and cochlear implants: an expanded cohort and etiologic assessment. Otol Neurotol 34(3): 422-428.

85. Zhou G, Kenna MA, Stevens K, Licameli G (2009) Assessment of saccular function in children with sensorineural hearing loss. Arch Otolaryngol Head Neck Surg 135(1): 40-44.

86. Lin BY, Young YH (2016) Assessing residual vestibular function in adults with congenital hearing loss. Eur Arch Otorhinolaryngol 273(12): 42094214 .

87. Zhou Y, Wu Y, Wang J (2016) Otolithic organ function in patients with profound sensorineural hearing loss. J Otol 11(2): 73-77.

88. Ledesma AL, Barreto MA, Bahmad F (2014) Vestibular evoked myogenic potential: its use in Sudden Sensorineural Hearing Loss. Int Tinnitus J 19(1): 82-5.

89. Iversen MM, Rabbitt RD (2020) Biomechanics of Third Window Syndrome. Front Neurol 11: 891.

90. Merchant SN, Rosowski JJ (2008) Conductive hearing loss caused by third-window lesions of the inner ear. Otol Neurotol 29(3): 282-289.

91. Wackym PA, Balaban CD, Zhang P, Siker DA, Hundal JS (2019) Third Window Syndrome: Surgical Management of Cochlea-Facial Nerve Dehiscence. Front Neurol 10: 1281.

92. Ungar OJ, Handzel 0, Cavel O, Oron Y (2018) Superior semicircular canal dehiscence with concomitant otosclerosis-A literature review and case discussion. Clin Case Rep 6(12): 2364-2370.

93. Maxwell AK, Slattery WH, Gopen QS, Miller ME (2020) Failure to close the gap: Concomitant superior canal dehiscence in otosclerosis patients. Laryngoscope 130(4): 1023-1027. 
94. Fernandez Ignacio J, Molinari Giulia, Presutti Livio (2020) Decision Making in Patients with Concomitant Otosclerosis and Superior Semicircular Canal Dehiscence. Otology \& Neurotology 42(1): e1-e9.

95. Ahmed W, Rajagopal R, Lloyd G (2019) Systematic Review of Round Window Operations for the Treatment of Superior Semicircular Canal Dehiscence. J Int Adv Otol 15(2): 209-214.

96. Silverstein H, Kartush JM, Parnes LS, Poe DS, Babu SC, et al. (2014) Round window reinforcement for superior semicircular canal dehiscence: a retrospective multi-center case series. Am J Otolaryngol 35(3): 286-93.

97. Succar EF, Manickam PV, Wing S, Walter J, Greene JS, et al. (2018) Round window plugging in the treatment of superior semicircular canal dehiscence. Laryngoscope 128(6): 1445-1452.

98. Plonka PM, Passeron T, Brenner M, Tobin DJ, Shibahara S, et al. (2009) What are melanocytes really doing all day long...? Exp Dermatol 18(9):799-819.

99. Gick B, Derrick D (2009) Aero-tactile integration in speech perception. Nature 462: 502-504.

100. Do-Hyoung Kim, Chan-Young Choi, Chae-Heuck Lee, Mee Joo (2015) Primary Intracranial Leptomeningeal Melanomatosis. J Korean Neurosurg Soc 58(6): 554-556.

101. Sara SJ, Bouret S (2012) Orienting and reorienting: the locus coeruleus mediates cognition through arousal. Neuron 76(1): 130-41.

102. Schlessinger DI, Anoruo M, Schlessinger J. Biochemistry, Melanin (2020) In: StatPearls [Internet]. Treasure Island (FL): StatPearls Publishing.

103. McCorry LK (2007) Physiology of the autonomic nervous system. Am J Pharm Educ 71(4): 78.

104. Eddie Perkins, Susan Warren, Paul J May (2009) The Mesencephalic Reticular Formation as a Conduit for Primate Collicular Gaze Control: Tectal Inputs to Neurons Targeting the Spinal Cord and Medulla. Anat Rec (Hoboken) 292(8): 1162-1181.

105. Manzoni D, O Pompeiano, C D Barnes, G Stampacchia, P d'Ascanio (1989) Convergence and interaction of neck and macular vestibular inputs on locus coeruleus and subcoeruleus neurons. Pflugers Arch. 413: $580-598$.

106. Wilson VI, Maeda M (1974) Connections between semicircular canals and neck motorneurons in the cat. J Neurophysiol 37(2): 346-57.

107. Masazumi Masuda, Kazuto Yamazaki, Jin Kanzaki, Yasuhiro Hosoda (1994) Ultrastructure of melanocytes in the dark cell area of human vestibular organs: Functional implications of gap junctions, isolated cilia, and annulate lamellae.

108. Ruth Gussen (1978) Round Window Niche Melanocytes and Webby Tissue. Possible collateral route of vascular and perilymph circulation Arch Otolaryngol 104(11): 662-668.

109. Benson JC, Diehn F, Passe T, Guerin J, Silvera VM, et al. (2020) The Forgotten Second Window: A Pictorial Review of Round Window Pathologies. AJNR Am J Neuroradiol 41(2): 192-199.
110. Qunfeng Cai, Carolyn Whitcomb, Jessica Eggleston, Wei Sun, Richard Salvi, et al. (2013) Round window closure affects cochlear responses to supra-threshold stimuli. Laryngoscope 123(12): E116-E121.

111. Yetişer S, Kertmen M (2007) Cochlear conductive hearing loss in patients with Meniere's disease. Kulak Burun Bogaz Ihtis Derg 17(1): 18-21.

112. Ravi R Shah, James G Naples, Michael J Ruckenstein, Steven J Eliades (2019) Sudden sensorineural hearing loss as a presenting symptom in superior semicircular canal dehiscence.

113. Kiumehr S, Mahboubi H, Djalilian HR (2012) Posterior semicircular canal dehiscence following endolymphatic sac surgery. Laryngoscope 122(9): 2079-2081.

114. Wendt WJ, Hashikawa A (2019) Enlarged Vestibular Aqueduct Syndrome: Sudden Hearing Loss in a Child with a Cerebral Shunt. Pediatr Emerg Care 35(7): e135-e137.

115. Ellis M. Arjmand, Audra Webber BS (2004) Audiometric Findings in Children with a Large Vestibular Aqueduct. Arch Otolaryngol Head Neck Surg 130(10): 1169-1174.

116. Zekveld AA, Koelewijn T, Kramer SE (2018) The Pupil Dilation Response to Auditory Stimuli: Current State of Knowledge. Trends Hear 22: 2331216518777174.

117. Wong K, Kozin ED, Kanumuri VV, Vachicouras N, Miller J, et al. (2019) Auditory Brainstem Implants: Recent Progress and Future Perspectives. Front Neurosci 13: 10

118. Dieter A, Keppeler D, Moser T (2020) Towards the optical cochlear implant: optogenetic approaches for hearing restoration. EMBO Mol Med 12(4): e11618.

119. Grohé C, Lee B, Flynn JJ (2018) Recent inner ear specialization for high-speed hunting in cheetahs. Sci Rep 8: 2301.

120. Kaelin CB, Xu X, Hong LZ, David VA, McGowan KA, et al. (2012) Specifying and sustaining pigmentation patterns in domestic and wild cats. Science 337(6101): 1536-41.

121. Saeed HK, Al-Abbasi AM, Al-Maliki SK, Al-Asadi JN (2018) Sensorineural hearing loss in patients with chronic renal failure on hemodialysis in Basrah, Iraq. Ci Ji Yi Xue Za Zhi 30(4): 216-220.

122. Tan HE, Lan NSR, Knuiman MW, Divitini ML, Swanepoel DW, et al. (2018) Associations between cardiovascular disease and its risk factors with hearing loss-A cross-sectional analysis. Clin Otolaryngol 43(1): 172-181

123. Iverson J, Goldin-Meadow S (1998) Why people gesture when they speak. Nature 396: 228.

124. Kolarik AJ, Cirstea S, Pardhan S, Moore BC (2014) A summary of research investigating echolocation abilities of blind and sighted humans. Hear Res 310: 60-68. 